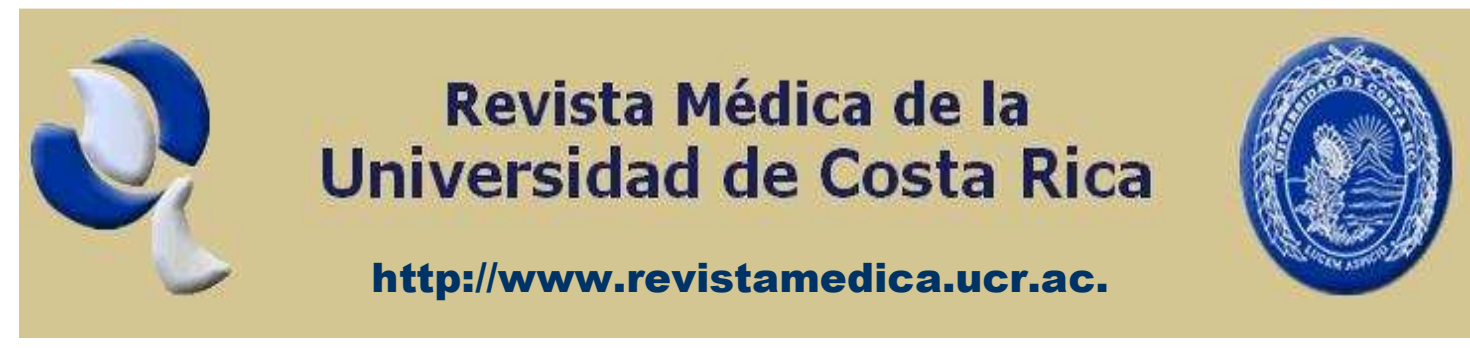

Investigación original

\title{
Resistencia antimicrobiana en bacterias del grupo Bacteroides fragilis aisladas a partir de muestras diarreicas de pacientes pediátricos y geriátricos costarricenses.
}

\author{
Quirós Quesada, Miguel ${ }^{1}$; Rodríguez Sánchez, César ${ }^{1}$ y Rodríguez Cavallini, \\ Evelyn".
}

1. Laboratorio de Investigación en Bacteriología Anaerobia y Centro de Investigación en Enfermedades Tropicales. Universidad de Costa Rica, San Pedro, San José, Costa Rica.

Resumen: Se determinó el perfil de susceptibilidad de 13 aislamientos del grupo Bacteroides fragilis, recuperados en medios selectivos diferenciales a partir de 6 de 31 muestras diarreicas de niños y 3 de 8 muestras de ancianos hospitalizados, mediante un sistema comercial y la técnica de microdilución en caldo. Tres aislamientos de niños presentaron concentraciones mínimas inhibitorias (CMI) de penicilina mayores a $256 \mu \mathrm{g} \mathrm{ml}^{-1}$ y varios aislamientos fueron resistentes a mezclas de $\beta$ lactámicos con inhibidores de $\beta$-lactamasas. La resistencia a cefalosporinas se documentó mediante el hallazgo de dos aislamientos de niños con CMI de de 128 $\mu \mathrm{g}$ de cefotaxime $\mathrm{ml}^{-1}$. Un aislamiento de niños y dos de ancianos exhibieron CMI de clindamicina mayores o iguales a $32 \mathrm{\mu g} \mathrm{ml}^{-1}$, en tanto que dos cepas de niños y una de ancianos se asociaron con CMI de metronidazol mayores a $128 \mathrm{\mu g} \mathrm{ml}^{-1} \mathrm{o}$ de $16 \mu \mathrm{g} \mathrm{ml}^{-1}$, respectivamente. Se observaron algunas discrepancias entre los resultados de sensibilidad antimicrobiana obtenidos por ambos métodos. El conocimiento de esta resistencia es relevante porque algunas de estas drogas son muy utilizadas en Costa Rica y porque las especies del grupo $B$. fragilis pueden fungir como un reservorio de genes de resistencia a nivel intestinal.

Revista electrónica publicada por el Departamento de Farmacología de la Escuela de

Medicina de la Universidad de Costa Rica, 2060 San José, Costa Rica. ® All rights

reserved. 
Palabras clave: Bacteroides fragilis, resistencia antimicrobiana, biota intestinal, concentración mínima inhibitoria

Recibido: Julio 2008. Aceptado: Agosto 2008. Publicado: Septiembre 2008.

Abstract: This manuscript reports the susceptibility pattern of 13 isolates of the Bacteroides fragilis group recovered from 6 of 31 diarrheic samples of infants and 3 of 8 samples of elderly people. All patients analyzed were admitted in two Costa Rican hospitals. To obtain these results, bacteria were cultivated on selective media and their antibiotic susceptibility was determined using both a commercial system and a broth microdilution method. Three isolates of children exhibited minimum inhibitory concentrations (MIC) of penicillin above $256 \mathrm{\mu g} \mathrm{ml}^{-1}$. In addition, several isolates tolerated combinations of $\beta$-lactam antibiotics with $\beta$ lactamase inhibitors. The finding of two isolates of children with $\mathrm{CMI}$ of $128 \mu \mathrm{g}$ of cefotaxime $\mathrm{ml}^{-1}$ exemplified the occurrence of cephalosporin-resistant bacteria. One isolate of children and two of elderly patients were associated with CMI of clindamycin equal or higher than $32 \mathrm{\mu g} \mathrm{ml}^{-1}$. On the other hand, two isolates of children and one of elderly patients had CMI of metronidazole higher than $128 \mu \mathrm{g}$ $\mathrm{ml}^{-1}$ or $16 \mu \mathrm{g} \mathrm{ml}^{-1}$, respectively. Some discrepancies between the results obtained with the commercial system and the broth microdilution method were observed. The information herein summarized is of relevance because some of the drugs studied are commonly prescribed in Costa Rica and also because the species of the Bacteroides fragilis group may serve as a reservoir of resistance genes in the gut ecosystem.

Keywords: Bacteroides fragilis, minimum inhibitory concentrations (MIC), antimicrobial resistance.

\section{Introducción}

El grupo Bacteroides fragilis incluye bacilos Gram-negativos, anaerobios, sacarolíticos, resistentes a la bilis, que profusamente habitan el colon y, en menor grado, la cavidad oral y el tracto respiratorio superior del ser humano [1]. Las 10 especies de este grupo, a decir, $B$ caccae, $B$. distasonis, B. eggerttii, $B$. merdae, $B$. ovatus, $B$. stercoris, $B$. el citoesqueleto de las alteración de la permeabilidad de las uniones intercelulares de los thetaiotaomicrom, $B$. uniformis, $B$. vulgatus y $B$. fragilis constituyen el principal tipo de bacterias anaerobias Gram-negativas que se suele aislar a partir de muestras clínicas $[1,2]$. En lo que a diarreas se refiere, algunas cepas de $B$. fragilis producen una metaloproteinasa dependiente de zinc que altera

células intestinales, provoca enterocitos y la salida de líquido al lumen intestinal $[1,3]$.

Revista electrónica publicada por el Departamento de Farmacología de la Escuela de 
La literatura documenta el papel de las especies del grupo $B$. fragilis en la transferencia lateral de genes de resistencia a otras bacterias intestinales $\quad[4,5,6]$ Para contextualizar este fenómeno en nuestro medio es necesario iniciar por conocer el perfil de susceptibilidad de aislamientos de individuos que podrían llegar a recibir terapia antimicrobiana. Esto por cuanto el uso de antibióticos representa una presión selectiva para el establecimiento y la diseminación de genes de resistencia adquirida [7].

En este trabajo determinamos la presencia de bacterias del grupo $B$. fragilis en muestras diarreicas de 31 niños y 8 ancianos hospitalizados mediante cultivo en medios selectivos diferenciales $y$ determinamos su patrón de susceptibilidad a diferentes antibióticos usando un sistema comercial y la técnica de microdilución en caldo.

Considerando que la mayoría de las infecciones por anaerobios son de origen endógeno y que el grupo $B$. fragilis es el que con mayor frecuencia las produce, el conocimiento de la resistencia antimicrobiana se torna relevante no solo por el peligro potencial que podría representar para el paciente, sino también por el papel que las especies de este grupo juega en la diseminación de genes de resistencia a nivel intestinal.

\section{Materiales y Métodos}

Se colectaron 39 muestras diarreicas de pacientes internados en los hospitales Nacional de Niños (HNN) y Geriátrico Raúl Blanco Cervantes
(HGRBC) durante un período de 12 meses. Se incluyeron en el estudio muestras de pacientes cuyo cuadro diarreico se presentó al menos 48 horas después del ingreso al centro de salud. Las 31 muestras de niños (HNN 1-31) y 8 de ancianos (HGRBC 1-8) fueron congeladas a $-70^{\circ} \mathrm{C}$ y transportadas en hielo seco hasta el Laboratorio de Investigación en Bacteriología Anaerobia de la Universidad de Costa Rica. Una asada de cada muestra fue rayada directamente sobre platos de agar Bacteroides-Bilis-Esculina (BBE) y Sangre Lisada-KanamicinaVancomicina (LKV) que fueron incubados en jarra de anaerobiosis a 35ํㅡ por 48 horas [8]. Concluido este periodo se procedió a realizar una tinción de Gram y una prueba de tolerancia al oxígeno a todas aquellas colonias circulares, convexas y de borde entero de más de $1 \mathrm{~mm}$ de diámetro, color café oscuro en BBE o amarillentas en LKV [9]. Los aislamientos en cultivo puro fueron identificados con el sistema miniaturizado rapid ID $32 A^{\circledR}$ de acuerdo con las instrucciones brindadas por el fabricante (BioMérieux). En este contexto, la cepa Bacteroides fragilis ATCC 23745 fue utilizada como control de crecimiento y de identificación. La susceptibilidad de aislamientos asignados a especies del grupo $B$. fragilis a diferentes antimicrobianos fue estudiada con el sistema ATB ANA $^{\circledR}$ siguiendo las recomendaciones del fabricante (BioMérieux). Simultáneamente se determinó la concentración mínima inhibitoria (CMI) de penicilina, clindamicina, cloranfenicol, metronidazol (Sigma

Revista electrónica publicada por el Departamento de Farmacología de la Escuela de 
Chemical Co.), imipenem (Merck \& Co.) y cefotaxime (Karnataka Antibiotics \& Pharmaceuticals Limited) con el método de microdilución en caldo [10]. La única modificación al método oficial fue inocular los platos con una suspensión ajustada al estándar 1 de Mc Farland a fin de lograr el número de colonias deseadas en los platos de control sin necesidad de usar una cámara de anaerobiosis. Como control de calidad se utilizaron las cepas B. fragilis ATCC 25285 y B. thetaiotaomicron ATCC 29741. Del análisis de expedientes se pudo concluir que las muestras HNN3 y HNN24 fueron positivas por Clostridium difficile productor de toxina $A$ y que la muestra HNN23 incluyó un Clostridium perfringens enterotoxigénico. En ningún caso se detectó Salmonella sp., Shigella sp. o rotavirus y solamente una de las muestras provenía de un paciente que había sido tratado con antibióticos (amikacina, HGRBC2).

\section{Resultados y discusión}

Se aislaron 13 bacterias del grupo $B$. fragilis a partir de 9 de las 39 muestras analizadas: 8 a partir de muestras pediátricas y 5 a partir de muestras geriátricas (Cuadro 1).

En concordancia con otros reportes [11], la resistencia a penicilinas con o sin inhibidores de $\beta$-lactamasas fue común. Así, tres aislamientos de niños presentaron $\mathrm{CMI}$ mayores a $256 \mu \mathrm{g} \mathrm{ml} l^{-1}$ y varios aislamientos fueron resistentes a mezclas de $\beta$ lactámicos con inhibidores de $\beta$ lactamasas (Cuadros 2 y 3 ). Esto abre la posibilidad de que mecanismos adquiridos de resistencia tales como la metalo- $\beta$ lactamasa cfiA [12] estén presentes en la colección aquí estudiada. Tal y como ha sido comprobado en otros estudios [13,14], ningún aislamiento fue resistente a la combinación piperacilina-tazobactam ni al imipenem. La resistencia a cefalosporinas de segunda (cefoxitina y cefotetán) y de tercera generación (cefotaxime) también fue manifiesta (Cuadros 2 y 3). Aquí resalta el hallazgo de dos aislamientos de niños con CMl de $128 \mu \mathrm{g}$ de cefotaxime $\mathrm{ml}^{-}$ ${ }^{1}$; fenotipo que podría ser explicado por la actividad de la cefalosporinasa cromosomal CepA [15].

La resistencia a clindamicina se ha documentado desde hace varios años en varias áreas geográficas $[16,17,18,19]$. Cuatro de los 15 aislamientos (27\%) fueron categorizados como resistentes a esta droga por el método de ATB $\mathrm{ANA}^{\circledR}$, mientras que cinco de ellos lo fueron por el método de microdilución (Cuadro 3). Un aislamiento de niños y dos de ancianos dieron lugar a CMI mayores o iguales a $32 \mathrm{\mu g} \mathrm{ml}^{-1}$. Esto es particularmente importante debido a que esta droga es utilizada frecuentemente en Costa Rica. En este caso la resistencia a clindamicina podría ser conferida por metilasas adquiridas, tal y como ha sido documentado en otros estudios [20].

Otra discrepancia se detectó en las determinaciones de susceptibilidad a metronidazol. En detalle, solamente una de las cepas con CMI elevadas fue categorizada por el ATB ANA ${ }^{\circledR}$ como resistente mientras que dos cepas de niños y una de ancianos se

Revista electrónica publicada por el Departamento de Farmacología de la Escuela de 
asociaron con CMl mayores a $128 \mu \mathrm{g}$ $\mathrm{ml}^{-1}$ y de $16 \mathrm{\mu g} \mathrm{ml}^{-1}$, respectivamente (Cuadro 3). Debido a que el método de de referencia es el de microdilución, los tres aislamientos se consideraron resistentes; este fenotipo podría deberse a la presencia de nitroreductasas nim [21]. En nuestro medio, el metronidazol es utilizado para combatir infecciones por bacterias anaerobias y como antiparasitario, por lo que su uso es frecuente.

Si bien es cierto que en ninguna de las muestras puede atribuirse a cepas de Bacteroides sp. la etiología de la diarrea, el hallazgo de CMI muy elevadas en pacientes susceptibles debería contribuir al uso racional de los antibióticos. Esto por cuanto es sabido que las bacterias de este género pueden transferir determinantes de resistencia antimicrobiana beta-lactámicos, clindamicina y metronidazol a otras bacterias intestinales.

Cuadro 1.

Identificación y origen de los aislamientos del grupo Bacteroides fragilis aislados de muestras diarreicas de pacientes pediátricos y geriátricos costarricenses

\begin{tabular}{|c|c|c|}
\hline Aislamiento & Muestra de origen & Identificación \\
\hline $\begin{array}{l}\text { B1 } \\
\text { B2 } \\
\text { B3 } \\
\text { B4 } \\
\text { B5 } \\
\text { B6 } \\
\text { B7 } \\
\text { B8 } \\
\text { B9 } \\
\text { B10 } \\
\text { B13 } \\
\text { B14 } \\
\text { B15 }\end{array}$ & $\begin{array}{l}\text { HNN-3 } \\
\text { HNN-3 } \\
\text { HNN-5 } \\
\text { HNN-5 } \\
\text { HNN-23 } \\
\text { HNN-24 } \\
\text { HNN-25 } \\
\text { HNN-29 } \\
\text { HGRBC-2 } \\
\text { HGRBC-2 } \\
\text { HGRBC-6 } \\
\text { HGRBC-7 } \\
\text { HGRBC-7 }\end{array}$ & $\begin{array}{c}\text { B. uniformis } \\
\text { B. fragilis } \\
\text { B. fragilis } \\
\text { B. uniformis } \\
\text { B. ovatus } \\
\text { B. eggerthii } \\
\text { B. uniformis } \\
\text { B. eggerthii } \\
\text { B. uniformis } \\
\text { B. ovatus } \\
\text { B. eggerthii } \\
\text { B. thetaiotaomicron } \\
\text { B. ovatus }\end{array}$ \\
\hline
\end{tabular}

Revista electrónica publicada por el Departamento de Farmacología de la Escuela de Medicina de la Universidad de Costa Rica, 2060 San José, Costa Rica. ® All rights reserved. 


\section{Cuadro 2.}

Patrón de sensibilidad de aislamientos del grupo B. fragilis provenientes de muestras diarreicas de pacientes pediátricos y geriátricos costarricenses según el método ATB-ANA

\begin{tabular}{|c|c|c|c|c|c|c|c|c|}
\hline \multicolumn{9}{|c|}{ Antimicrobianos (rango de concentraciones ensayadas en $\mu \mathrm{g} \mathrm{ml}^{-1}$ )* } \\
\hline $\begin{array}{c}\text { Aislamient } \\
0\end{array}$ & $\begin{array}{c}\text { Amoxicilin } \\
\quad \begin{array}{l}\text { a } \\
2-4\end{array}\end{array}$ & $\begin{array}{c}\text { Amoxicilina } \\
/ \\
\text { Ác. } \\
\text { clavulánico } \\
8 / 4\end{array}$ & $\begin{array}{c}\text { Piperacilin } \\
\text { a }\end{array}$ & $\begin{array}{c}\text { Piperacilina } \\
/ \\
\text { tazobactam } \\
\\
64 / 4\end{array}$ & $\begin{array}{l}\text { Ticarcilin } \\
\text { a }\end{array}$ & $\begin{array}{c}\text { Ticarcilina/ } \\
\text { Ác. } \\
\text { clavulánic } \\
0 \\
64 / 2\end{array}$ & $\begin{array}{c}\text { Cefoxitin } \\
\text { a }\end{array}$ & $\begin{array}{c}\text { Cefotetá } \\
\mathrm{n}\end{array}$ \\
\hline B1 & $R$ & $R$ & $R$ & $S$ & $R$ & $S$ & $S$ & $R$ \\
\hline B2 & $\mathrm{R}$ & $\mathrm{R}$ & $\mathrm{R}$ & $S$ & $\mathrm{R}$ & $S$ & $S$ & $\mathrm{R}$ \\
\hline B3 & $\mathrm{R}$ & $S$ & $\mathrm{R}$ & $S$ & $\mathrm{R}$ & $S$ & $S$ & $S$ \\
\hline B4 & $\mathrm{R}$ & $\mathrm{R}$ & $\mathrm{R}$ & $S$ & $\mathrm{R}$ & $S$ & $S$ & $\mathrm{R}$ \\
\hline B5 & $\mathrm{R}$ & $S$ & $\mathrm{R}$ & $S$ & $R$ & $S$ & $S$ & $R$ \\
\hline B6 & $R$ & $S$ & $R$ & $S$ & $R$ & $S$ & $S$ & $R$ \\
\hline B7 & $S$ & $S$ & $S$ & $S$ & $S$ & $S$ & $S$ & $R$ \\
\hline B8 & $R$ & $S$ & $S$ & $S$ & $S$ & $S$ & $S$ & $R$ \\
\hline B9 & $R$ & $S$ & $S$ & $S$ & $S$ & $S$ & $S$ & $R$ \\
\hline B10 & $\mathrm{R}$ & $S$ & $S$ & $S$ & $S$ & $S$ & $\mathrm{R}$ & $R$ \\
\hline $\mathrm{B} 13$ & $S$ & $S$ & $S$ & $S$ & $S$ & $S$ & $S$ & $S$ \\
\hline B14 & $S$ & $S$ & $S$ & $S$ & $\mathrm{~S}$ & $S$ & $S$ & $\mathrm{R}$ \\
\hline B15 & $\mathrm{R}$ & $S$ & $S$ & $S$ & $S$ & $S$ & $S$ & $\mathrm{R}$ \\
\hline
\end{tabular}

*Los resultados de penicilina, cefotaxime, imipenem, clindamicina, cloranfenicol y metronidazol están implícitos en el Cuadro 3.

Revista electrónica publicada por el Departamento de Farmacología de la Escuela de Medicina de la Universidad de Costa Rica, 


\section{Cuadro 3.}

Concentraciones mínimas inhibitorias y categorización de aislamientos del grupo $B$. fragilis cultivados a partir de muestras diarreicas de pacientes pediátricos y geriátricos costarricenses.

\begin{tabular}{|c|c|c|c|c|c|c|c|c|c|c|c|c|}
\hline \multicolumn{13}{|c|}{ CMI ( $\mu \mathrm{g} / \mathrm{ml})$ e interpretación ${ }^{*}$} \\
\hline \multirow[t]{2}{*}{ Aislamiento } & \multicolumn{2}{|c|}{ Penicilina } & \multirow{2}{*}{\multicolumn{2}{|c|}{$\begin{array}{l}\text { Cefotaxime } \\
\text { CMI }\end{array}$}} & \multicolumn{2}{|c|}{ Imipenem } & \multicolumn{2}{|c|}{ Clindamicina } & \multicolumn{2}{|c|}{ Cloranfenicol } & \multicolumn{2}{|c|}{ Metronidazo } \\
\hline & CMI & & & & CMI & & CMI & & CMI & & CMI & \\
\hline B1 & 32 & $\mathrm{R}$ & 32 & $\mathrm{R}$ & $<1$ & $\mathrm{~S}$ & $<1$ & $\mathrm{~S}$ & 4 & $\mathrm{~S}$ & $<4$ & $\mathrm{~S}$ \\
\hline B2 & $\geq 256$ & $\mathrm{R}$ & 128 & $\mathrm{R}$ & $<1$ & $\mathrm{~S}$ & 4 & $\mathrm{R}$ & 8 & $\mathrm{~S}$ & 8 & $\mathrm{~S}$ \\
\hline B3 & $\geq 256$ & $\mathrm{R}$ & $<8$ & $\mathrm{~S}$ & $<1$ & $\mathrm{~S}$ & 4 & $\mathrm{R}$ & 4 & $\mathrm{~S}$ & $<4$ & $\mathrm{~S}$ \\
\hline B4 & $\geq 256$ & $\mathrm{R}$ & 128 & $\mathrm{R}$ & $<1$ & $\mathrm{~S}$ & 2 & $S$ & 4 & $S$ & $<4$ & $\mathrm{~S}$ \\
\hline B5 & 8 & $\mathrm{R}$ & $<8$ & $S$ & $<1$ & $S$ & $<1$ & $S$ & 8 & $S$ & $\geq 128$ & R \\
\hline B6 & 64 & $\mathrm{R}$ & 64 & $\mathrm{R}$ & $<1$ & $S$ & $\geq 32$ & $\mathrm{R}$ & 8 & $S$ & $<4$ & $S$ \\
\hline B7 & 16 & $\mathrm{R}$ & $<8$ & $\mathrm{~S}$ & $<1$ & $S$ & $<1$ & $S$ & 8 & $S$ & $\geq 128$ & $\mathrm{R}$ \\
\hline B8 & 8 & $\mathrm{R}$ & $<8$ & $S$ & $<1$ & $\mathrm{~S}$ & $<1$ & $\mathrm{~S}$ & $<2$ & $S$ & $<4$ & $\mathrm{~S}$ \\
\hline B9 & 8 & $\mathrm{R}$ & 32 & $\mathrm{R}$ & $<1$ & $S$ & $\geq 32$ & $\mathrm{R}$ & 4 & $\mathrm{~S}$ & $<4$ & $\mathrm{~S}$ \\
\hline B10 & 16 & $\mathrm{R}$ & $<8$ & $\mathrm{~S}$ & $<1$ & $S$ & $\geq 32$ & $\mathrm{R}$ & 4 & $\mathrm{~S}$ & $<4$ & $S$ \\
\hline B13 & 16 & $\mathrm{R}$ & $<8$ & $S$ & $<1$ & $S$ & 2 & $S$ & 4 & $\mathrm{~S}$ & $<4$ & $S$ \\
\hline B14 & 8 & $\mathrm{R}$ & $<8$ & $S$ & $<1$ & $S$ & $<1$ & $S$ & $<2$ & S & 16 & $\mathrm{R}$ \\
\hline B15 & 16 & $\mathbf{R}$ & 32 & $\mathbf{R}$ & $<1$ & $\mathrm{~S}$ & 2 & $\mathrm{~S}$ & 8 & $\mathrm{~S}$ & $<4$ & $\mathrm{~S}$ \\
\hline
\end{tabular}

${ }^{*}$ Los resultados incongruentes con el método de ATB ANA ${ }^{\circledR}$ aparecen en negrita ${ }^{\text {R: }}$ resistente; S: sensible

Revista electrónica publicada por el Departamento de Farmacología de la Escuela de Medicina de la Universidad de Costa Rica, 2060 San 


\section{Agradecimientos}

Al señor Pablo Vargas por su ayuda técnica y a la Vicerrectoría de Investigación de la Universidad de Costa Rica por el financiamiento brindado mediante el Proyecto 803A5-027.

\section{Referencias}

1. Wexler, H.M. Bacteroides: the good, the bad, and the nitty-gritty. Clinical Microbiological Reviews, october 2007; 20(4):593-621.

2. Rodríguez, E., Gamboa, M.M., Rodríguez, C. \& Vargas, P. Grupo Bacteroides fragilis en heces humanas no diarreicas y su sensibilidad antimicrobiana. Revista Española de Quimioterapia, diciembre 2006; 19(4):357-362.

3. Obiso, R.J., Lyerly, D.M., van Tassell, R.L. \& Wilkins T. Proteolytic activity of the Bacteroides fragilis enterotixin causes fluid secretion and the intestinal damage in vivo. Infection and Immunity, october 1995; 63(10):3820-3826.

4. Salyers A., Moon, K., \& Schlesinger, D. The human intestinal tract - a hotbed of resistance gene transfer? Part I. Clinical Microbiology Newsletter, 2007; 29(3).

5. Salyers, A., Gupta, A. \& Wang, Y. Human intestinal bacteria as reservoirs for antibiotic resistance genes. Trends in Microbiology, september 2004; 12(9):412-416.

6. Martínez, J.L., \& Barquero, F. Interactions among strategies associated with bacterial infection: pathogenicity, epidemicity, and antibiotic resistance. Clinical Microbiology Reviews, october 2002; 15(4):647-679.

7. Friedman, C.R. \& Whitney, C.G. It's time for a change in practice: reducing antibiotic use can alter antibiotic resistance. Journal of Infectious Diseases, april 2008; 197(8):1082-1083.
8. Jousimies-Somer, H.R., Sumannen, P., Citron, D.M., et al. Wadsworth Anaerobic Bacteriology Manual. Star Publishing Co., Belmont, United States. 2002, pp287.

9. Rodríguez, E. \& Quesada, C. Bacteriología Anaerobia: principios y diagnóstico de laboratorio. Lara Segura \& Asociados. San José, Costa Rica. 2008, pp88-110.

10. Hecht, D.W., Aldrigde, K.E., Citron, D.M., et al. NCCLS. Methods for antimicrobial susceptibility testing of anaerobic bacteria; Approved Standard$6^{\text {th }}$ Edition. NCCLS document M11-A6. 2004, pp1-41.

11. Jousimies-Somer, H.R., Summanen, P.H., Finegold, S.M., et al. Bacteroides, Porphyromonas, Prevotella, Fusobacterium, and other anaerobic Gram-negative bacteria. In: Manual of Clinical Microbiology Vol. 1. Murray, P.R., et. al. (eds.) ASM Press, Washington D.C., United States. $8^{\text {th }}$ edition 2003, pp880-901.

12. ESCMID study group on antimicrobial resistance in anaerobic bacteria, Soki, J., Edwards, R., et. al. Examination of cfiA-mediated carbapenem resistance in Bacteroides fragilis strains from a European antibiotic susceptibility survey. International Journal of Antimicrobial Agents, december 2006; 28(6):497-502.

13. Aldridge, K.E., \& O'Brien, M. In vitro susceptibilities of the Bacteroides fragilis group species: change in isolation rates

Revista electrónica publicada por el Departamento de Farmacología de la Escuela de 
